\title{
Voluntary Wheel Running Exercise Improves Aging-Induced Sarcopenia via Activation of Peroxisome Proliferator-Activated Receptor Gamma Coactivator-1a/Fibronectin Type III Domain- Containing Protein 5/Adenosine Monophosphate-Activated Protein Kinase Signaling Pathway
}

\author{
Young Jun $\mathrm{Ko}^{1}$, Il-Gyu $\mathrm{Ko}^{2}$ \\ ${ }^{1}$ Major in Sport Service practice, College of Welfare Convergence, Kangnam University, Youngin, Korea \\ ${ }^{2}$ Department of Physiology, College of Medicine, Kyung Hee University, Seoul, Korea
}

Purpose: In this study, the protective effect of voluntary wheel running exercise on muscle loss and muscle weakness in gastrocnemius of old rats was investigated. The association of voluntary wheel exercise with the peroxisome proliferator-activated receptor gamma coactivator- $1 \alpha$ (PGC-1 $\alpha$ )/fibronectin type III domain-containing protein 5 (FNDC5)/adenosine monophosphate-activated protein kinase (AMPK) signaling pathway and vascular endothelial growth factor (VEGF) expression was also evaluated.

Methods: Six-month-old and 22-month-old male rats were used for this experiment. The rats in voluntary wheel running exercise groups were performed wheel running for 2 months. Weight bearing test for walking strength, rotarod test for motor coordination and balance, hematoxylin and eosin (H\&E) staining for histological changes in the muscle tissues, Western blot analysis for PGC-1a, FNDC5, AMPK, immunofluorescence for VEGF were conducted.

Results: Decreased muscle mass, strength, and coordination due to aging were associated with a decrease in the PGC-1a/ FNDC5/AMPK signaling pathway in the gastrocnemius. Voluntary wheel running exercise enhanced VEGF expression by activating the PGC-1 $\alpha$ /FNDC5/AMPK signaling pathway, then increased muscle mass, strength, and coordination.

Conclusions: It has been suggested that voluntary wheel running exercise alleviates symptoms of urological diseases that are difficult to treat. Wheel running exercise is a good therapeutic strategy to prevent or treat aging-related sarcopenia.

Keywords: Aging; Sarcopenia; Voluntary wheel running exercise; Muscle mass; Strength

- Fund/Grant Support: This study was approved by the Institutional Care and use Committee of Kyung Hee University (KHUASP[SE]-19-150).

- Conflict of Interest: No potential conflict of interest relevant to this article was reported.

\footnotetext{
- HIGHLIGHTS

- Decreased muscle mass, strength, and coordination due to aging were associated with a decrease of PGC-1a/FNDC5/AMPK signaling pathway.

- Voluntary wheel running exercise enhanced VEGF expression by activating PGC-1a/FNDC5/AMPK signaling pathway.

- Wheel running exercise increased muscle mass, strength, and coordination.
}

Corresponding author: Il-Gyu Ko (iD) https://orcid.org/0000-0003-2696-6869 Department of Physiology, College of Medicine Kyung Hee University, 26 Kyungheedae-ro, Dongdaemun-gu, Seoul 02447, Korea Email: rhdlfrb@hanmail.net

Submitted: March 2, 2021 / Accepted after revision: April 24, 2021
This is an Open Access article distributed under the terms of the Creative Commons Attribution Non-Commercial License (http://creativecommons.org/licenses/by-nc/4.0/) which permits unrestricted non-commercial use, distribution, and reproduction in any medium, provided the original work is properly cited. 


\section{INTRODUCTION}

Aging is a complex phenomenon that causes organ dysfunction as tissue damage accumulates. One of the tissues most affected by aging is skeletal muscle. Sarcopenia is a degenerative skeletal muscle condition in which muscle mass and function are significantly reduced [1]. Sarcopenia is considered one of the health threats to an aging population due to its association with weakness, immobility, hospitalization, and death [2].

Several targeting factors are being considered to improve aging-induced sarcopenia, and in previous studies, peroxisome proliferator-activated receptor gamma coactivator-1a (PGC-1 $\alpha$ ) has emerged as a major factor $[3,4]$. In skeletal muscle, PGC-1 $\alpha$ is known to regulate mitochondrial biogenesis and function, and is generally regulated by adenosine monophosphate-activated protein kinase (AMPK) [5]. In addition to regulating muscle mitochondrial function, PGC-1 1 a plays a greater role in regulating proteolysis and autophagy, neuromuscular junctions, macrophage/inflammatory responses, and necrosis $[3,6]$. Overexpression of skeletal muscle PGC-1 1 improves strength, endurance, and motor function in aged mice [7].

Physical exercise is known to affect autophagy and mitophagy to prevent loss of muscle mass and disruption of mitochondria due to aging. Lifelong exercise prevented age-related decrease in the mRNA expression of PGC-1 $\alpha$, p53, and p21 [8]. PGC-1 $\alpha$ allows muscle mitochondria to better cope with high lipid loads, reflecting the underlying metabolic effect of exercise training [9]. Upregulation of PGC-1 $\alpha$ in muscles improved exercise performance and increased maximal oxygen uptake in various exercise paradigms [10]. Exercise potently increased PGC-1 $\alpha$ expression in muscle, and PGC-1 $1 \alpha$ overexpression in skeletal muscle significantly increased endurance by activating mitochondria oxidation and angiogenesis [4].

Wheel running exercise is known to improve motor function and prevent physical weakness. Wheel running in rodents is more similar to the natural running of a person than to a forced treadmill running [11]. Wheel running exercise is used to assess physical performance and improve health. Unlike other experimental models relying on aversive stimuli to force active movement, wheel running is a voluntary activity, [12]. Exercise is known to help improve muscle strength and motor coordination with balance [13].

In the current study, the protective effect of voluntary wheel running exercise on muscle loss and weakness in gastrocnemius of old rats was investigated. The association of voluntary wheel exercise with the PGC-1 $\alpha /$ fibronectin type III domaincontaining protein 5 (FNDC5)/AMPK signaling pathway and vascular endothelial growth factor (VEGF) expression was also evaluated.

\section{MATERIALS AND METHODS}

\section{Animals and Grouping}

Six-month old male Fischer F344 rats $(\mathrm{n}=16,290 \pm 10 \mathrm{~g})$ and 22-month-old rats ( $\mathrm{n}=16,380 \pm 10 \mathrm{~g}$ ) were purchased from the Orient Bio Co. (Seongnam, Korea). The rats were classified into one of 4 groups ( $n=8$ in each group): young-age group, youngage and wheel running exercise group, old-age group, old-age and wheel running exercise group. We received approval number (KHUASP[SE]-19-150) from the Kyung Hee University Institutional Animal Care and Use Committee.

\section{Wheel Running Exercise Protocol}

After stabilizing the experimental animals, they performed wheel running for 2 months. The rats of the wheel running exercise groups were individually placed in cages (diameter, 20 $\mathrm{cm}$; width, $9 \mathrm{~cm}$ ) equipped with running wheel. The mileage was calculated as the number of rotations of the wheel using an electronic sensor attached to the driving wheel (average daily mileage: $227.68 \pm 17.80 \mathrm{~m}$ ).

\section{Weight Bearing Test}

In order to measure walking strength, the weight bearing test was done 4 times at 2-week intervals according to the previously described method [14]. The weight applied to each limb during voluntary walking was measured with a weight bearing test devise. The device was configured to monitor the weight bearing of the rat leg at up to 4 different points along the route. The bottom of the path consisted of 8 acrylic plates $(5 \mathrm{~cm} \times 10 \mathrm{~cm})$ attached to the load cell (work range 0-1,000 g, Dacell Co., Cheongwon, Korea). As the laboratory animals walked and applied pressure to the floor, the output of each load cell was fed to a digital amplifier (Axon Instruments Inc., Union City, NJ, USA) for proper amplification and filtering. The processed signals were calculated using software (Cambridge Electronic Design, Cambridge, UK) and expressed as a percentage with each weight applied.

\section{Rotarod Test}

The latency of the rotarod test for motor coordination and bal- 
ance was measured according to the method described below [13]. The rotarod test was done 8 times at 10-day interval during the wheel running exercise. Each rat was placed in a separate compartment on a rotating rod (diameter, $7 \mathrm{~cm} ; 30 \mathrm{rpm}$ fixed velocity) of a rotarod devise (Biological Research Apparatus, Ugo Basile, Varese, Italy). The latency until fall was automatically recorded, and we limited the rat's latency to $300 \mathrm{sec}-$ onds to eliminate stress and fatigue.

\section{Tissue Preparation}

Tissue preparation was done according to the previously described method $[15,16]$. The gastrocnemius was removed after anesthesia with Zoletil 50 (10 mg/kg; Vibac Laboratories). The extracted muscles were measured by wet weight and the ratio of muscle weight to body weight was calculated. After the muscle tissues were harvested, the muscle tissues were treated with $4 \%$ paraformaldehyde and 70\%, 80\%, 90\%, 95\%, 100\% ethanol, incubated with xylene, then embedded in paraffin. Microtome (Thermo Fisher Scientific, Waltham, MA, USA) was used to make the gastrocnemius tissue into 5 - $\mu \mathrm{m}$-thick slide ( 6 sections per muscle tissue), and the slides were dried overnight at $37^{\circ} \mathrm{C}$ on a hot plate.

\section{Hematoxylin and Eosin Staining}

Hematoxylin and Eosin (H\&E) staining was done according to the previously described method $[17,18]$. The slides were incubated with Harris hematoxylin for 10 seconds, washed, treated with eosin (Sigma Aldrich Co., St. Louis, MO, USA) for $5 \mathrm{sec}-$ onds, and washed again. After drying, the slides were dehydrated by soaking in ethanol and xylene, and sealed with Permount (Thermo Fisher Scientific). Images were captured using an Image Analysis System (Leica Microsystems, Wetzlar, Germany).

\section{Western Blotting}

Western blot analysis was done according to the previously described method $[19,20]$. The gastrocnemius tissues were homogenized on chilled radioimmunoprecipitation assay buffer (RIPA buffer) (Cell Signaling Technology, Inc., Danvers, MS, USA), and then centrifuged at $14,000 \mathrm{rpm}$ for 30 minutes at $4^{\circ} \mathrm{C}$. Protein contents were measured using a $\mu$-drop reader (Thermo Fisher Scientific). The primary and secondary antibodies used were shown in Table 1. After reaction of primary and secondary antibodies, the blot membranes were measured using enhanced chemiluminescence detection kit (Bio-Rad, Hercules, CA, USA). Bands were measured by Image-Pro plus computer-assisted image analysis system (Media Cyberbetics Inc., Silver Spring, MD, USA), and the control was set to 1.00 for relative comparison.

\section{Immunofluorescence}

Immunofluorescence was done according to the previously described method $[15,20]$. Paraffin slides containing muscle tissues were deparaffinized with xylene and graded ethanol for 5 minutes, and washed with deionized water for 5 minutes. The slides were boiled in $10 \mathrm{mM}$ sodium citrate buffer for 2 minutes at $95^{\circ} \mathrm{C}$. Subsequently, the slides were cooled for 30 minutes at room temperature, and blocked with phosphate buffered saline containing $5 \%$ normal goat serum. The tissue sections were reacted overnight at $4^{\circ} \mathrm{C}$ with a 1:200 dilution of mouse antiVEGF antibody. After washing the primary anti-VEGF antibody, the slides were treated with a secondary Alexa 488-antimosue IgG antibody (1:500 dilution; Thermo Fisher Scientific) for 2 hours at room temperature. The slides were mounted on a coverslip using Vectashield and fluorescence images were captured by the Leica DMi8 fluorescence microscope (Leica Microsystems) wavelength in the excitation $490 \mathrm{~nm}$ to emission $525 \mathrm{~nm}$ for Alexa 488.

Table 1. Primary and secondary antibody used in Western blot

\begin{tabular}{lcccl}
\hline Classification & Items & Source & Titer & Company \\
\hline Primary antibody & PGC-1a & Anti-mouse & $1: 1,000$ & Santa Cruz Biotechnology, Santa Cruz, CA, USA \\
& FNDC5 & Anti-rabbit & $1: 1,000$ & Abcam, Cambridge, UK \\
Secondary antibody & AMPK, p-AMPK & Anti-rabbit & $1: 1,000$ & Cell Signaling Technology, Inc., Danvers, MS, USA \\
& HRP-conjugated IgG & Mouse & $1: 2,000$ & Vector Laboratories, Burlingame, CA, USA \\
& Rabbit & & \\
& & & \\
\hline
\end{tabular}

PGC-1 $\alpha$, peroxisome proliferator-activated receptor gamma coactivator-1 $\alpha$; FNDC5, fibronectin type III domain-containing protein 5; p-AMPK, phosphorylation adenosine monophosphate-activated protein kinase; HRP, horseradish peroxidase. 


\section{Statistical Analysis}

IBM SPSS Statistics ver. 23.0 (IBM Co., Armonk, NY, USA) was used and statistical analysis was done by 1-way analysis of variance followed by Duncan posttest. Data were expressed as the mean \pm standard error of the mean, and $\mathrm{P}<0.05$ was set statistically significant.

\section{RESULTS}

\section{Walking Strength and Motor Coordination}

The results of walking strength and motor coordination obtained in the weight bearing test and rotarod test were shown in Fig. 1. As a result, the old rats showed a decrease in walking strength and motor coordination compared to the young rats $(\mathrm{P}<0.05)$. On the other hand, the wheel running exercise alleviated the decrease in walking strength and motor coordination due to aging $(\mathrm{P}<0.05)$.

\section{Muscle Weight and Histology}

Weight and histological changes of gastrocnemius were shown in Fig. 2. Histological analysis showed that aging caused atrophy of muscle fibers and a marked increase in connective tissues compared to rats of young age. These changes decreased the ratio of gastrocnemius to body weight $(\mathrm{P}<0.05)$. However, wheel running exercise increased muscle in the gastrocnemius of the aged rats $(\mathrm{P}<0.05)$.

\section{PGC-1a/FNDC5/AMPK Signaling Pathway}

Western blot analysis was used to investigate the effect of wheel running on PGC-1 $\alpha$ and FNDC5 activation and AMPK phosphorylation (Fig. 3). Aging induced a decrease in AMPK phosphorylation, resulting in decreased PGC-1 $\alpha$ and FNDC5 expression in the gastrocnemius compared to young-age rats $(\mathrm{P}<0.05)$. On the other hand, wheel running exercise increased AMPK phosphorylation and PGC-1 $\alpha$ and FNDC5 expression in the gastrocnemius of old rats $(\mathrm{P}<0.05)$.

\section{VEGF Expression}

VEGF expression in sarcopenic rats according to age was shown in Fig. 4. In aging-induced sarcopenia, the expression of VEGF in the gastrocnemius was decreased, but the wheel running exercise increased the expression of VEGF in the gastrocnemius of old rats.

\section{DISCUSSION}

Muscle strength is related to walking ability, and coordination is also a motor function that can protect the body from accidents such as fall. Older people need a higher amount of load each week than younger people to maintain the muscle fiber hypertrophy achieved during the gradual training program [21]. Aging-induced decreased myocyte proliferation and increased myostatin mRNA and protein expression decreased muscle mass and muscle strength [14]. However, treadmill exercise improved muscle mass and strength by increasing muscle cell proliferation and inhibiting myostatin mRNA and protein expression [14]. Histological findings in old rats in the current study showed muscle fiber atrophy compared to younger rats.

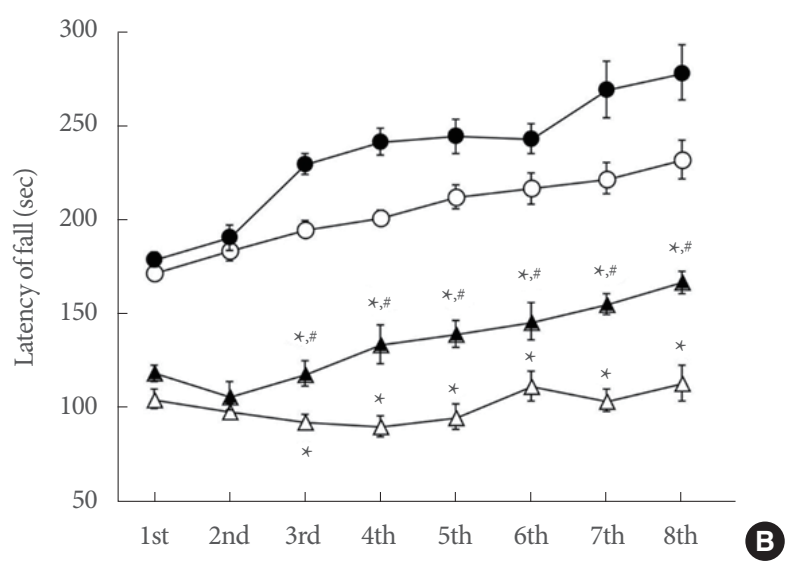

Fig. 1. Walking strength and motor coordination. (A) Walking strength in the weight bearing test. (B) Motor coordination in the rotarod test. $\bigcirc$, young-age group; $\bullet$, young-age wheel running exercise group; $\triangle$, old-age group; $\boldsymbol{\Delta}$, old-age wheel running exercise group. ${ }^{\star} \mathrm{P}<0.05$ compared to young-age group. ${ }^{*} \mathrm{P}<0.05$ compared to old-age group. 

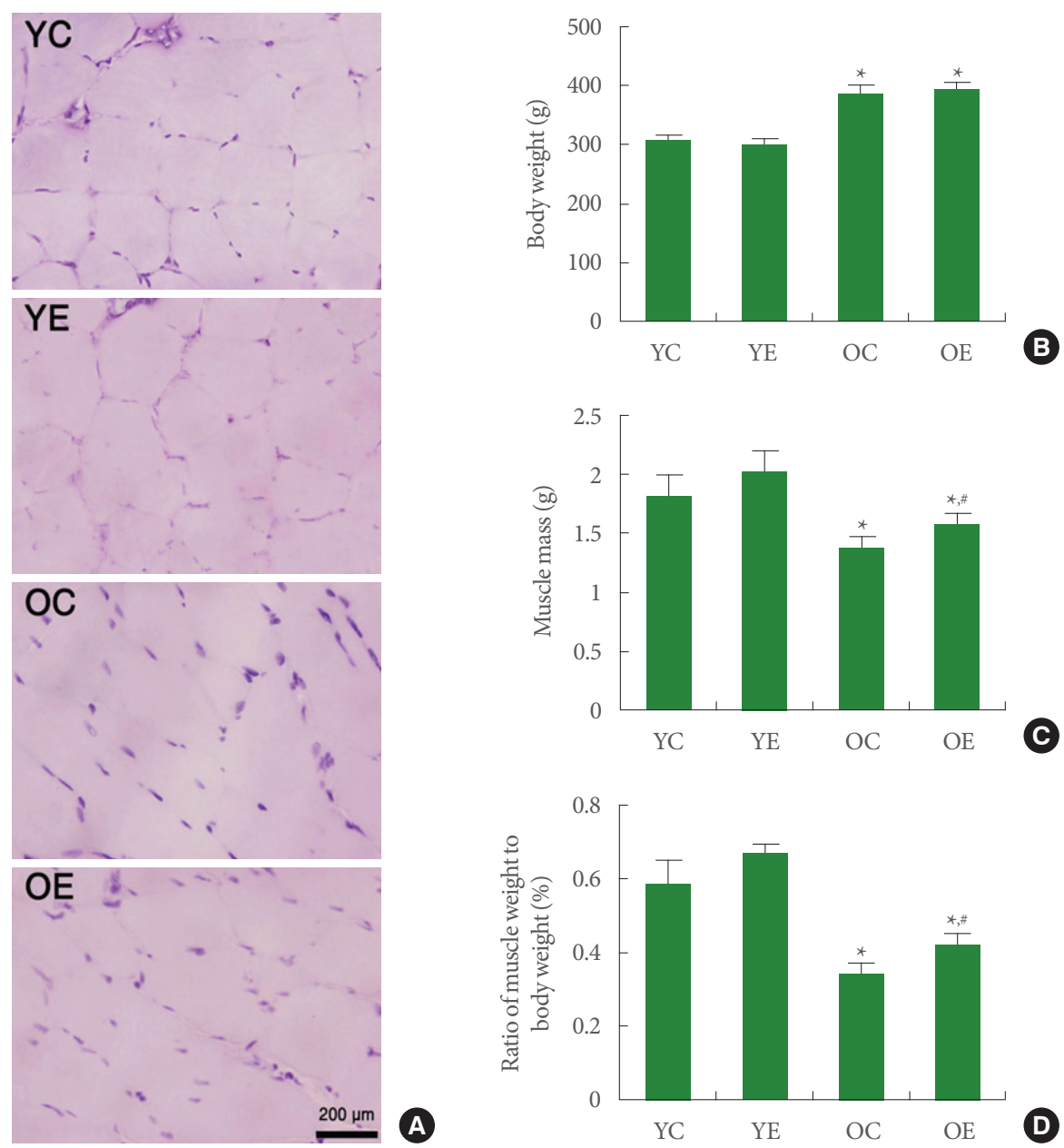

Fig. 2. Changes of histological evaluation and muscle weight. (A) Photomicrographs of sarcopenia. The sections were stained with hematoxylin (blue is myocytic nucleus) and eosin (pink is muscle fiber). (B) Body weight in each group. (C) Gastrocnemius weight in each group. (D) Ratio of body weight to gastrocnemius weight. YC, young-age group; YE, young-age wheel running exercise group; OC, old-age group; $\mathrm{OE}$, old-age wheel running exercise group. ${ }^{*} \mathrm{P}<0.05$ compared to young-age group. ${ }^{*} \mathrm{P}<0.05$ compared to old-age group.

In addition, walking strength in the weight bearing test and motor coordination in the rotarod test were reduced in the old rats.

There are several factors associated with muscle mass loss and weakness as a result of the aging process. Among them, PGC-1a regulates mitochondrial biosynthesis and improves muscle atrophy [3]. PGC-1 $\alpha$ mediates gene regulation in skeletal muscle adaptation in the old people [4,7]. Exercise enhanced serum irisin, skeletal muscle FNDC5 (irisin precursor), and its upstream activator PGC-1 $\alpha$ [22]. Irisin is a myokine regulated by PGC-1 $\alpha$ in exercising skeletal muscle and is released into the bloodstream after cleavage of FNDC5. An increase in irisin through an increase in FNDC5 changes the morphology of brown adipose tissue and stimulates angiogenesis for muscle cell proliferation [23]. For this reason, PGC-1 1 , which promotes muscle hypertrophy, may be a therapeutic target to prevent muscle wasting due to aging. In the current study, agingrelated sarcopenia decreased PGC-1 $\alpha$, resulting in decreased FNDC5 expression. The decrease in FNDC5 eventually suppressed the expression of VEGF in the gastrocnemius. These results indicated that age-related deficiency of PGC-1 $\alpha$ and FNDC5 exacerbated sarcopenia by reducing VEGF.

PGC-1 $\alpha$ is required to prevent age-related decrease in citrate synthase and superoxide dismutase in skeletal muscle [24]. Activation of the AMPK/PGC-1 $\alpha$ signaling cascade is implicated in preventing skeletal muscle atrophy [25]. Impaired mitochon- 

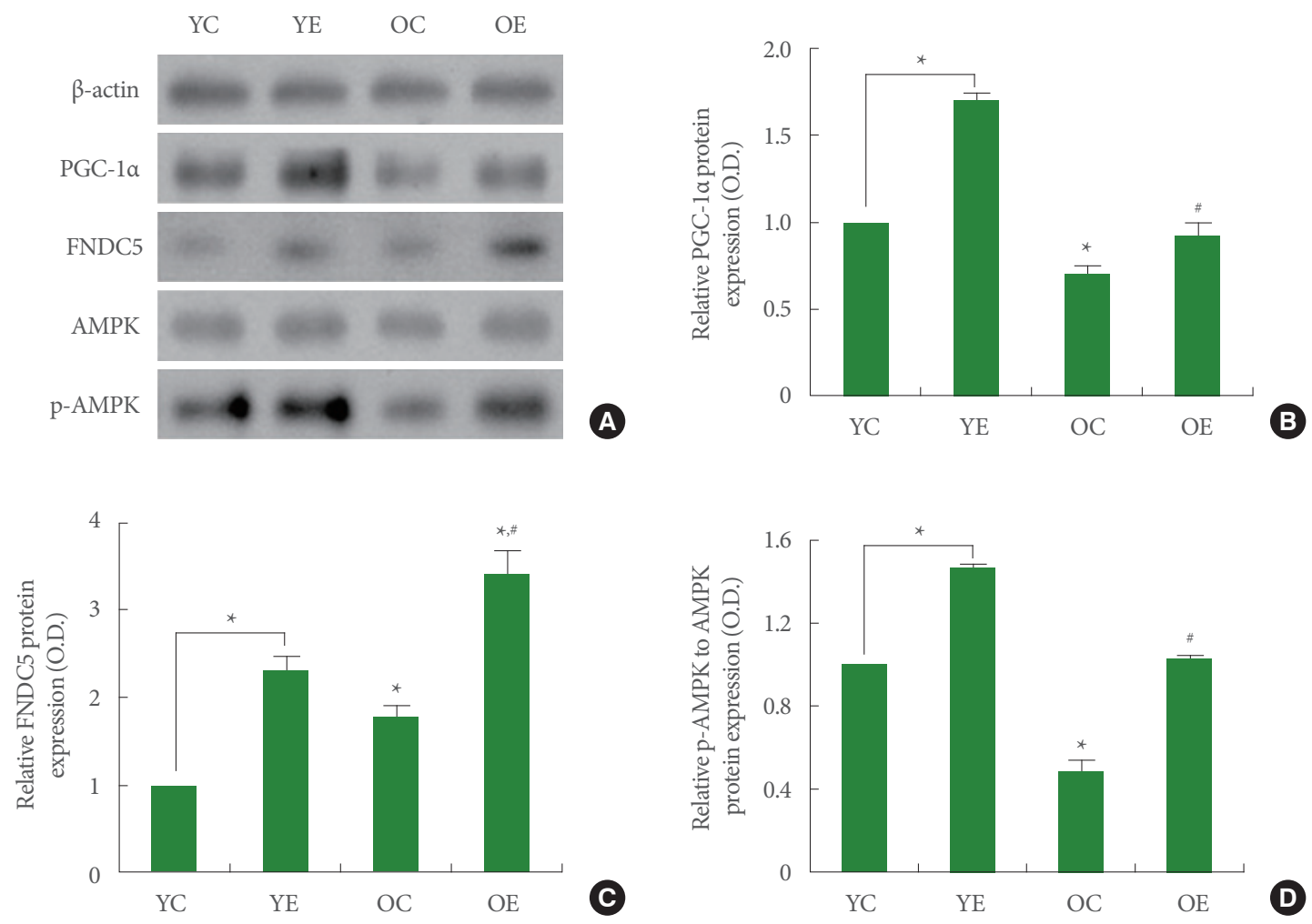

Fig. 3. Expression of peroxisome proliferator-activated receptor gamma coactivator-1a (PGC-1a)/fibronectin type III domain-containing 5 (FNDC5)/adenosine monophosphate-activated protein kinase (AMPK) signaling pathway. (A) Representative expression of PGC-1 $\alpha$, FNDC5, AMPK, phosphorylated AMPK (p-AMPK). (B) PGC-1a expression in each group. (C) FNDC5 expression in each group. (D) p-AMPK expression in each group. YC, young-age group; YE, young-age wheel running exercise group; OC, old-age group; $\mathrm{OE}$, old-age wheel running exercise group. ${ }^{\star} \mathrm{P}<0.05$ compared to young-age group. ${ }^{*} \mathrm{P}<0.05$ compared to old-age group.

drial function and altered biological pathways lead to aging of skeletal muscles, causing to muscle atrophy and decreased muscle performance in the old people [26]. In the current study, voluntary wheel running exercise improved the expression of AMPK, which was reduced due to aging. These changes activated PGC-1 $\alpha$ and FNDC5, which were reduced by aging in skeletal muscle.

Acute exercise increased VEGF mRNA in human skeletal muscles, ultimately improving oxygen and energy substrate delivery to exercising muscles [27]. PGC-1 $\alpha$ is an essential element in the exercise-induced VEGF expression in skeletal muscle [28]. The expression of FNDC5 was regulated by the action of PGC-1a to increase VEGF [29]. VEGF exerted a neuroprotective effect and had a neurotrophic function, and voluntary exercise increased VEGF expression [30]. In the current study, AMPK activation due to wheel running exercise enhanced the expression of PGC-1 $\alpha$ and FNDC5 in aged rats. AMPK activation induced increment of VEGF expression in skeletal muscle.
Here in this study, wheel running exercise enhanced VEGF expression by activating the PGC-1a/FNDC5/AMPK signaling pathway, and then increased muscle mass, strength, and coordination. Wheel running exercise is a good treatment strategy for preventing or treating aging-induced sarcopenia.

\section{AUTHOR CONTRIBUTION STATEMENT}

- Conceptualization: $I G K$

- Data curation: YJK

- Formal analysis: YJK

- Funding acquisition: IGK

- Methodology: YJK

- Project administration: IGK

- Visualization: YJK

- Writing-original draft: IGK

- Writing-review \& editing: IGK 

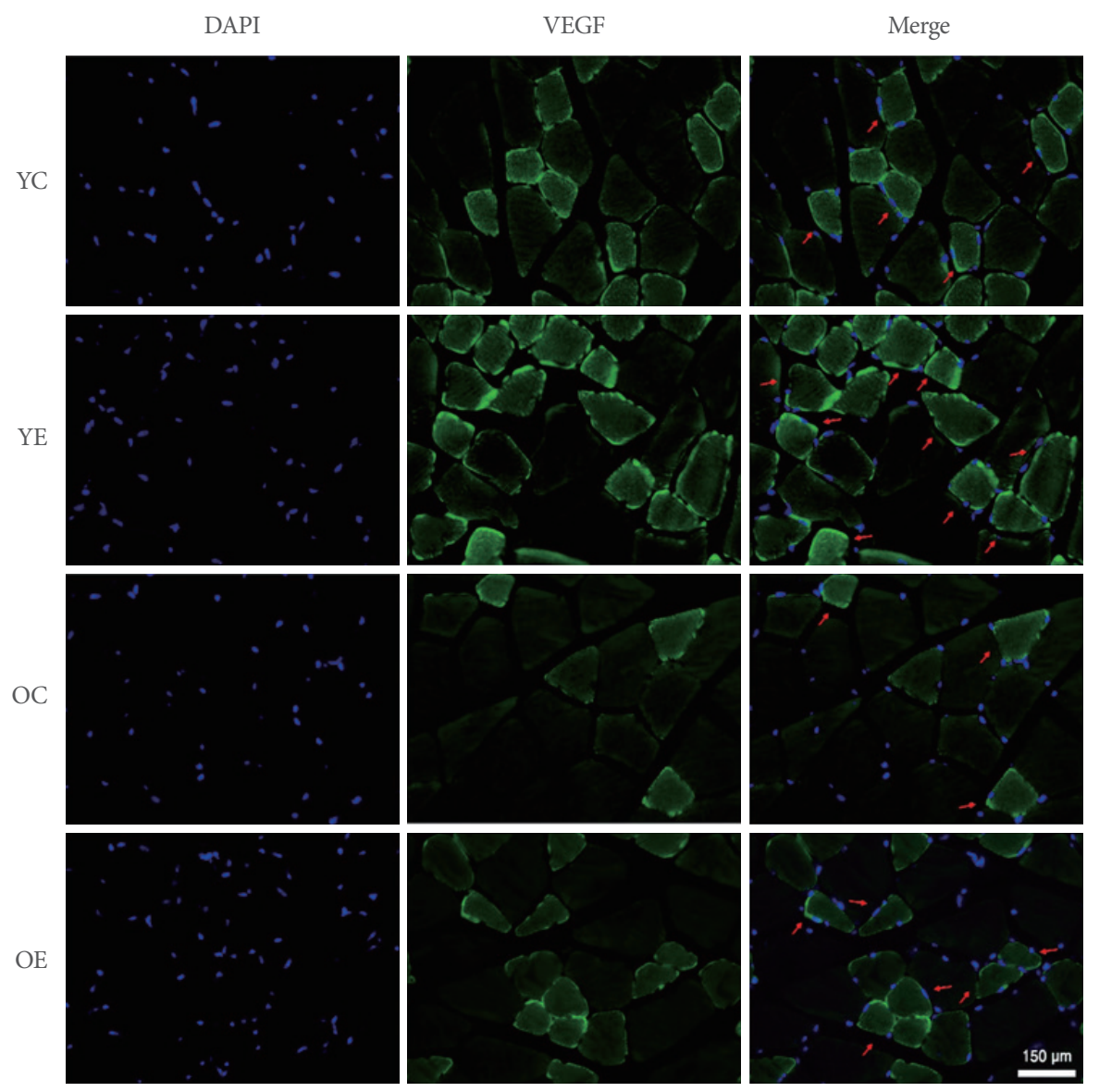

Fig. 4. Expression of vascular endothelial growth factor (VEGF) in the gastrocnemius tissue. Red arrows represent VEGF-positive cells. DAPI, 4'-6-diamidino-2-phenylindole; YC, young-age group; YE, young-age wheel running exercise group; OC, old-age group; OE, old-age wheel running exercise group.

\section{ORCID}

Young Jun Ko http://orcid.org/0000-0001-5469-7235

Il-Gyu Ko http://orcid.org/0000-0003-2696-6869

\section{REFERENCES}

1. Kinoshita H, Orita S, Inage K, Yamauchi K, Abe K, Inoue M, et al. Skeletal muscle cell oxidative stress as a possible therapeutic target in a denervation-induced experimental sarcopenic model. Spine 2019;44:446-55.

2. Ji LL, Kang C. Role of PGC-1 $\alpha$ in sarcopenia: etiology and potential intervention - a mini-review. Gerontology 2015;61:139-48.

3. Petrocelli JJ, Drummond MJ. PGC-1 1 -targeted therapeutic approaches to enhance muscle recovery in aging. Int J Environ Res Public Health 2020;17:8650.
4. Yang S, Loro E, Wada S, Kim B, Tseng WJ, Li K, et al. Functional effects of muscle PGC-1 $\alpha$ in aged animals. Skelet Muscle 2020; 10:14.

5. Jäger S, Handschin C, St-Pierre J, Spiegelman BM. AMP-activated protein kinase (AMPK) action in skeletal muscle via direct phosphorylation of PGC-1 $\alpha$. Proc Natl Acad Sci U S A 2007;104:1201722.

6. Cannavino J, Brocca L, Sandri M, Grassi B, Bottinelli R, Pellegrino MA. The role of alterations in mitochondrial dynamics and PGC1a over-expression in fast muscle atrophy following hindlimb unloading. J Physiol 2015;593:1981-95.

7. Gill JF, Santos G, Schnyder S, Handschin C. PGC-1a affects agingrelated changes in muscle and motor function by modulating specific exercise-mediated changes in old mice. Aging Cell 2018;17: e12697.

8. Dethlefsen MM, Halling JF, Møller HD, Plomgaard P, Regenberg B, 
Ringholm S, et al. Regulation of apoptosis and autophagy in mouse and human skeletal muscle with aging and lifelong exercise training. Exp Gerontol 2018;111:141-53.

9. Koves TR, Li P, An J, Akimoto T, Slentz D, Ilkayeva O, et al. Peroxisome proliferator-activated receptor- $\gamma$ co-activator $1 \alpha$-mediated metabolic remodeling of skeletal myocytes mimics exercise training and reverses lipid-induced mitochondrial inefficiency. J Biol Chem 2005;280:33588-98.

10. Calvo JA, Daniels TG, Wang X, Paul A, Lin J, Spiegelman BM, et al. Muscle-specific expression of PPAR $\gamma$ coactivator-1a improves exercise performance and increases peak oxygen uptake. J Appl Physiol 2008;104:1304-12.

11. Manzanares G, Brito-da-Silva G, Gandra PG. Voluntary wheel running: patterns and physiological effects in mice. Braz J Med Biol Res 2018;52:e7830.

12. Goh J, Ladiges W. Voluntary wheel running in mice. Curr Protoc Mouse Biol 2015;5:283-90.

13. Kim JY, Yi ES, Lee H, Kim JS, Jee YS, Kim SE, et al. Swimming exercise ameliorates symptoms of MOG-induced experimental autoimmune encephalomyelitis by inhibiting inflammation and demyelination in rats. Int Neurourol J 2020;24:S39-47.

14. Ko IG, Jeong JW, Kim YH, Jee YS, Kim SE, Kim SH, et al. Aerobic exercise affects myostatin expression in aged rat skeletal muscles: a possibility of antiaging effects of aerobic exercise related with pelvic floor muscle and urethral rhabdosphincter. Int Neurourol J 2014; 18:77-85.

15. Ko YJ, Kim BK, Ji ES. Treadmill exercise in obese maternal rats during pregnancy improves spatial memory through activation of phosphatidylinositol 3-kinase pathway in the hippocampus of rat pups. J Exerc Rehabil 2020;16:483-8.

16. Kim TW, Park SS, Kim BK, Sim YJ, Shin MS. Effects of sildenafil citrate on peripheral fatigue and exercise performance after exhaustive swimming exercise in rats. J Exerc Rehabil 2019;15:751-6.

17. Ferreira MC, Oliveira MX, Souza JI, Souza RA, Machado TPG, Santos AP. Effects of two intensities of treadmill exercise on neuromuscular recovery after median nerve crush injury in Wistar rats. J Exerc Rehabil 2019;15:392-400.

18. Lee S, Won KY, Joo S. Protective Effect of polydeoxyribonucleotide against CCl4-induced acute liver injury in mice. Int Neurourol J 2020;24:S88-95.

19. Rho JH, Ko IG, Jin JJ, Hwang L, Kim SH, Chung JY, et al. Polydeoxyribonucleotide ameliorates inflammation and apoptosis in Achilles tendon-injury rats. Int Neurourol J 2020;24:S79-87.
20. Ji ES, Kim YM, Ko YJ, Baek SS. Treadmill exercise in obese maternal rats during pregnancy improves short-term memory through neurogenesis in the hippocampus of rat pups. J Exerc Rehabil 2020;16:392-7.

21. Bickel CS, Cross JM, Bamman MM. Exercise dosing to retain resistance training adaptations in young and older adults. Med Sci Sports Exerc 2011;43:1177-87.

22. Tiano JP, Springer DA, Rane SG. SMAD3 negatively regulates serum irisin and skeletal muscle FNDC5 and peroxisome proliferator-activated receptor $\gamma$ coactivator 1- $\alpha$ (PGC-1 $\alpha$ ) during exercise. J Biol Chem 2015;290:7671-84.

23. Rodriguez Lanzi C, Perdicaro DJ, Gambarte Tudela J, Muscia V, Fontana AR, Oteiza PI, et al. Grape pomace extract supplementation activates FNDC5/irisin in muscle and promotes white adipose browning in rats fed a high-fat diet. Food Funct 2020;11:1537-46.

24. Leick L, Lyngby SS, Wojtaszewski JF, Pilegaard H. PGC-1a is required for training-induced prevention of age-associated decline in mitochondrial enzymes in mouse skeletal muscle. Exp Gerontol 2010;45:336-42.

25. Kou X, Li J, Liu X, Yang X, Fan J, Chen N. Ampelopsin attenuates the atrophy of skeletal muscle from d-gal-induced aging rats through activating AMPK/SIRT1/PGC-1a signaling cascade. Biomed Pharmacother 2017;90:311-20.

26. Joseph AM, Adhihetty PJ, Buford TW, Wohlgemuth SE, Lees HA, Nguyen LM, et al. The impact of aging on mitochondrial function and biogenesis pathways in skeletal muscle of sedentary high- and low-functioning elderly individuals. Aging Cell 2012;11:801-9.

27. Jensen L, Pilegaard H, Neufer PD, Hellsten Y. Effect of acute exercise and exercise training on VEGF splice variants in human skeletal muscle. Am J Physiol Regul Integr Comp Physiol 2004;287: R397-402.

28. Leick L, Hellsten Y, Fentz J, Lyngby SS, Wojtaszewski JF, Hidalgo J, et al. PGC-1a mediates exercise-induced skeletal muscle VEGF expression in mice. Am J Physiol Endocrinol Metab 2009;297:E92103.

29. Nwadozi E, Ng A, Strömberg A, Liu HY, Olsson K, Gustafsson T, et al. Leptin is a physiological regulator of skeletal muscle angiogenesis and is locally produced by PDGFR $\alpha$ and PDGFR $\beta$ expressing perivascular cells. Angiogenesis 2019;22:103-15.

30. Lee MC, Byun K, Kim JS, Lee H, Kim K. Trends in exercise neuroscience: raising demand for brain fitness. J Exerc Rehabil 2019;15: 176-9. 